\title{
Tunability and Fano Resonance Properties in Different Types of One-Dimensional Superconductor Photonic Crystals
}

\author{
Arafa H. Aly * (D, D. Mohamed ${ }^{a}$, Z. S. Matar ${ }^{b}$, Y. Trabelsi ${ }^{c, d}$, D. Vigneswaran ${ }^{e}$, Fatima Tayeboun ${ }^{f}$, \\ M. A. Mohaseb ${ }^{a, b}$ \\ ${ }^{a}$ Beni-Suef University, Physics Department, TH-PPM group, Beni Suef, Egypt \\ ${ }^{b}$ Umm-Al-Qura University, Faculty of Applied Science, Department of Physics, Mecca, Saud Arabia \\ ${ }^{c}$ King Khalid University, College of Arts and Sciences in Muhail Asir, Physics Department, Abha, Saudi \\ Arabia \\ ${ }^{d}$ University of Tunis El Manar, National Engineering School of Tunis, Photovoltaic and Semiconductor \\ Materials Laboratory, 1002, Tunis, Tunisia \\ eSri Krishna College of Technology, Department of ECE, Coimbatore, 641 042, India \\ fUniversity Djillali Liabes, Faculty of technology, 22000, Sidi-Bel-Abbes Algeria
}

Received: November 08, 2020; Revised: March 03, 2021; Accepted: April 27, 2021

\begin{abstract}
The Fano resonance and EIR properties in different topological one-dimensional superconductor photonic crystals has been investigated theoretically using the Transfer Matrix Method (TMM). Different types of periodic heterostructures are studied and they are designed by alternating pairs of superconductor materials such ( $\mathrm{Nb} / \mathrm{BSCCO}),\left(\mathrm{Rb}_{3} \mathrm{C}_{60} / \mathrm{YBa}_{2} \mathrm{Cu}_{3} \mathrm{O}_{7}\right)$ and $\left(\mathrm{K}_{3} \mathrm{C}_{60} /(\mathrm{BiPb})_{2} \mathrm{Sr}_{2} \mathrm{Ca}_{2} \mathrm{Cu}_{3} \mathrm{O}_{\mathrm{y}}\right)$. All artificial periodic structures are sacked by dielectric cap layer at different induced fields. To exam the efficiency of the reported structures, different parameters are used for analysis such as layers thicknesses, temperature, angle of incidence, the kind of superconductor materials and the dielectric constant of the cap layer. The investigation results exhibit the presence of tunable Fano resonances and EIR resonance peak accompanied by asymmetrical line shape and they are very sensitive to the dielectric cap layer, the superconductor materials and the wave incidence angle.
\end{abstract}

Keywords: Tunability, Photonic crystals, Fano resonance.

\section{Introduction}

Photonic crystals (PCs) are the objects of various theoretical and experimental researches due to their ability to control the flow of electromagnetic waves (EMWs). Such microstructures open promising applications in modern optics $^{1-3}$ such as optical sensor ${ }^{4-6}$, laser LEDs ${ }^{7}$, optical microcavities $^{8}$, salinity ${ }^{9}$. These photonic band gap (PBG) materials with a periodic dielectric profile can be found in (1D), (2D) or (3D) dimension depending on kind of periodicity along the coordinate axes. A one-dimensional photonic crystal (1DPC) is the simplest form of PCs based on the modulation in layer along one dimension which favorable to the fabrication of integrated sub-micrometer optical device via various techniques like sol-gel process ${ }^{10}$. The transmittance spectra of PCs exhibit a switchable zones that prohibit and allow the propagation of electromagnetic waves (EMWs). The zone in which the propagating wave is forbidden are called photonic band gap (PBGs). The wave properties of these PBGs are sensitive to constituent materials and type of distributed layers along the propagative axe. In the past decades, the superconductor have introduced in photonic crystals due to their advantages and ability to reduce the damped EMWs instead of metal or normal materials. These

*e-mail: arafa.hussien@science.bsu.edu.eg new superconducting PCs provide an enlarged reflection bands can be tuned by the temperature of superconductor ${ }^{11-14}$. So, the response of system is mainly dependent on the London penetration depth, which is a function of the temperature and the external magnetic field as well ${ }^{15-17}$. Furthermore, the transmittance spectrum of considered superconducting PCs gives an asymmetric line-shape called Fano resonance and a quantum destructive interference phenomenon called Electromagnetic Induced Reflectance (EIR) ${ }^{18}$. The Fano resonance appears in PCs result on interference between a continuum band of state and a discrete quantum state and shows a sharp change of intensity light ${ }^{19,20}$. These Fano resonances given by superconducting PCs are originated of interesting applications in optical switching, sensing and filtering devices ${ }^{21-23}$. However, the tunable EIR is used specially for metamaterial and plasmonics ${ }^{24,25}$.

In this paper, we predict and demonstrate the generated Electromagnetic Induced Reflectance (EIR) and the Fano resonance of three different $1 \mathrm{D}$ periodic heterostructures. These photonic structures are composed by alternating diverse superconducting layers and finishing with a dielectric cap layers. The establish results have been investigated using the two-fluid model and the Transfer Matrix Method (TMM). Effect of the dielectric thickness, wave incidence angle, 
temperature degree, refractive index of dielectric cap layer and the principles of optical resonance are discussed.

\section{Theoretical Models}

In this section, we present the theoretical model of the proposed 1D PCs in the form of $(A B)^{N}$ delimited by dielectric cap layer. Here, layers A and B are set to be two different superconductor materials with thickness $d_{1}$ and $\mathrm{d}_{2}$ respectively. $\mathrm{N}$ the number of repetition of the pair (AB). Figure 1 displays the schematic diagram of the three proposed 1D periodic structures. Each structure consists of two different superconductor materials and limited by a dielectrics cap layer. The first structure consists of the pair superconductor layers ( $\mathrm{Nb} \backslash \mathrm{BSCCO})$. Whereas, the second one is $\left(\mathrm{Rb}_{3} \mathrm{C}_{60} \backslash \mathrm{YBa}_{2} \mathrm{Cu}_{3} \mathrm{O}_{7}\right)$ and the third structure consists of $\left(\mathrm{K}_{3} \mathrm{C}_{60} \backslash(\mathrm{BiPb}-2223)\right.$.

The Gorter-Casmir two fluid model is adopted to describe the electromagnetic response of the superconductor materials ${ }^{26,27}$. By adopting some approximations the relative permittivity of superconductor can be written as:

$\varepsilon_{\mathrm{s}}(\omega)=1-\frac{\mathrm{c}^{2}}{\omega^{2} \lambda_{\mathrm{L}}^{2}}$

Where, $\mathrm{c}$ and $\lambda_{\mathrm{L}}$ denote the velocity of light in vacuum and the temperature dependent London penetration depth ${ }^{26,27}$ noted as follows:
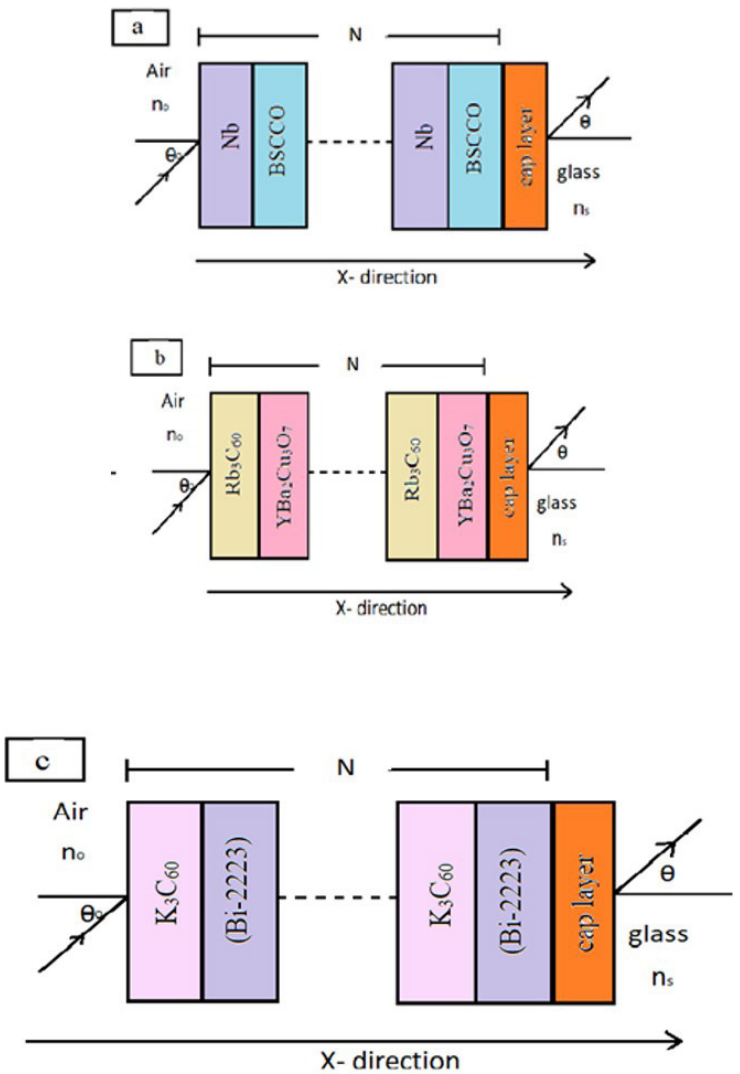

Figure 1. A Schematic diagram of the three proposed 1D periodic structures with different superconductor materials and delimited by dielectrics cap layers. Where a) $\mathrm{BSCCO}$; b) $\mathrm{YBa}_{2} \mathrm{Cu}_{3} \mathrm{O}_{7}$ and in c) Bi-2223.

$$
\lambda_{\mathrm{L}}=\frac{\lambda_{0}}{\sqrt{\left(1-\left(\frac{\mathrm{T}}{\mathrm{T}_{\mathrm{c}}}\right)^{\mathrm{q}}\right)}}
$$

With $\lambda_{\mathrm{o}}$ represent the penetration depth where $T=0 \mathrm{~K}$. Here $\mathrm{T}$ and $\mathrm{Tc}$ are the operating and the critical temperature of the superconducting materials. With q takes 2 for High Tc superconductivity and 4 for low Tc superconductivity. The interaction between the incidence EMWs and each interface layer through the periodic heterostructure is described using the following matrix ${ }^{27}$ :

$\mathrm{M}_{\mathrm{j}}=\left(\begin{array}{cc}\cos \left(\mathrm{k}_{\mathrm{j}} \delta_{\mathrm{j}}\right) & -\left(\mathrm{i} / \mathrm{p}_{\mathrm{j}}\right) \sin \left(\mathrm{k}_{\mathrm{j}} \delta_{\mathrm{j}}\right) \\ -\left(\mathrm{ip}_{\mathrm{j}}\right) \sin \left(\mathrm{k}_{\mathrm{j}} \delta_{\mathrm{j}}\right) & \cos \left(\mathrm{k}_{\mathrm{j}} \delta_{\mathrm{j}}\right)\end{array}\right)$

Where, $\mathrm{M}_{\mathrm{j}}$ is the characterized matrix of the $j^{\text {th }}$ layer. With $\delta_{\mathrm{j}}$ represents the phase variation at $\mathrm{j}^{\mathrm{t}}$ layer. For both TE and TM modes, $\delta_{\mathrm{j}}=\mathrm{d}_{\mathrm{j}} \mathrm{n}_{\mathrm{j}} \cos \theta_{\mathrm{j}}$, wheras, $\mathrm{p}_{\mathrm{j}}=\mathrm{n}_{\mathrm{j}} \cos \theta_{\mathrm{j}}$ for TM mode and $\mathrm{p}_{\mathrm{j}}=\cos \frac{\theta_{\mathrm{j}}}{\mathrm{n}_{\mathrm{j}}}$ for TM mode. ${ }^{\theta_{\mathrm{j}}}$ is the incidence angle.

The interaction between all stratified layers and incidence EMWs is described using the following matrix ${ }^{27}$ :

$M=\left(\begin{array}{ll}m_{11} & m_{12} \\ m_{21} & m_{22}\end{array}\right)=\left(M_{A} M_{B}\right)^{N}$

With $\mathrm{N}$ is the periodicity of the periodic superconducting layers. Thus, the transmittance values are given by the elements of transfer matrix $\mathrm{as}^{27}$ :

$t=\frac{2 p_{0}}{\left(M_{11}+M_{12} p_{f}\right) p_{0}+\left(M_{21}+M_{22} p_{f}\right)}$

With $\left(\mathrm{p}_{0, \mathrm{f}}=\sqrt{\frac{\varepsilon_{0}}{\mu_{0}}} \mathrm{n}_{0, \mathrm{f}} / \cos \theta_{0, \mathrm{f}}\right)$.

Then, the transmittance can be expressed $\mathrm{as}^{27}$ :

$\mathrm{T}=\frac{\mathrm{p}_{\mathrm{f}}}{\mathrm{p}_{0}}|\mathrm{t}|^{2}$

\section{Results and Discussion}

The concept of Fano and electromagnetic-induced reflectance resonances are recognized across multiple fields of physics due their interesting applications in optical switching, display, switching, nonlinear, and slow-light devices. The three heterostructures are studied in the visible and near infrared (IR) wavelength spectrum. The first heterostructure are designed using the pair of superconductor layers $\mathrm{N}_{\mathrm{b}}$ $\left(d_{1}=70 \mathrm{~nm}, T_{\mathrm{c}}=9.25 \mathrm{~K}, \lambda_{0}=83.4 \mathrm{~nm}^{28}\right)$, BSCCO as in Figure 1a, $\left(d_{2}=10 \mathrm{~nm}, T_{\mathrm{c}}=95 \mathrm{~K}, \lambda_{0}=150 \mathrm{~nm}^{29}\right)$. Whereas, the second one is made using $\mathrm{Rb}_{3} \mathrm{C}_{60}\left(d_{1}=70 \mathrm{~nm}, T_{\mathrm{c}}=30 \mathrm{~K}\right.$, $\left.\lambda_{0}=480 \mathrm{~nm}^{29}\right)$ and in Figure $1 \mathrm{~b}$ we used $\mathrm{YBa}_{2} \mathrm{Cu}_{3} \mathrm{O}_{7}\left(d_{2}=10 \mathrm{~nm}\right.$, $T_{\mathrm{c}}=93 \mathrm{~K}, \lambda_{0}=145 \mathrm{~nm}^{30}$ ) superconductor. The third structure consists of $\mathrm{K}_{3} \mathrm{C}_{60}\left(d_{1}=70 \mathrm{~nm}, T_{\mathrm{c}}=19.5 \mathrm{~K}, \lambda_{0}=420 \mathrm{~nm}^{31}\right)$ and in Figure $1 \mathrm{c}$ we used $(\mathrm{BiPb})_{2} \mathrm{Sr}_{2} \mathrm{Ca}_{2} \mathrm{Cu}_{3} \mathrm{O}_{\mathrm{y}}\left(d_{2}=10 \mathrm{~nm}\right.$, $T_{\mathrm{c}}=108 \mathrm{~K}, \lambda_{0}=150 \mathrm{~nm}^{32}$ ) superconductor materials. Where, 
$\mathrm{d}_{1,2}$, Tc and $\lambda_{\mathrm{L}}$ are the thicknesses, the critical temperature and the London penetration depth of consider superconductor. Let us consider all proposed $1 \mathrm{D}$ superconducting $\mathrm{PC}$ that are delimited by dielectric cap layered with $\left[\mathrm{n}_{3}=2.5, \mathrm{~d}_{3}=20 \mathrm{~nm}\right]$ and surrounded by air (In the right face) and glass substrate (In the left face).

Figure 2 shows the dependence of the reflectance on the normalized frequency of the three proposed heterostructures (a) $(\mathrm{Nb} / \mathrm{BSCCO})$, (b) $\left(\mathrm{Rb}_{3} \mathrm{C}_{60} / \mathrm{YBa}_{2} \mathrm{Cu}_{3} \mathrm{O}_{7}\right)$ and (c) $\left(\mathrm{K}_{3} \mathrm{C}_{60} /(\mathrm{BiPb})_{2} \mathrm{Sr}_{2} \mathrm{Ca}_{2} \mathrm{Cu}_{3} \mathrm{O}_{\mathrm{y}}\right)$. It is obvious that the all proposed heterostructure exhibit a similar spectrum with two symmetric narrow reflectance peaks. Figure 2 show two resonant peaks, in which the first represent the Fano resonance while the second having two side band is called the EIR. The first one called Fano resonance and the second peak with two side bands form a broad up ward reflectance valley called the EIR (Electromagnetic induced reflectance) that took place at 1.11 and 1.55 normalized frequencies.

Furthermore, the amplitude of the Fano resonance of the pair superconductors $(\mathrm{Nb} / \mathrm{BSCCO})$ reachs the full reflectance and it is higher than that of the $\left(\mathrm{Rb}_{3} \mathrm{C}_{60} / \mathrm{YBa} 2 \mathrm{Cu} 3 \mathrm{O} 7\right)$ and (c) $\left(\mathrm{K}_{3} \mathrm{C}_{60} /(\mathrm{BiPb})_{2} \mathrm{Sr}_{2} \mathrm{Ca}_{2} \mathrm{Cu}_{3} \mathrm{O}_{y}\right)$. However, the EIR reach to $70 \%$ of reflection for the two first pair superconducting PC structures and show an amplitude higher than that the third one. The given Fano resonance and EIR are due to the destructive interference of a discrete state with a continuum one. The proposed structures can serve as optical switching and light modulator.

Now, we investigated the influence of the wave incidence angle on the resonance properties of the three periodic photonic structures. Reflectance spectra versus incidence angles for TE polarization mode are shown in Figure 3. As the incidence angle increases, both Fano resonance and EIR shift toward the longer frequencies for the three structures. However, it is seen from the figures that this resonant peaks are strongly dependent to the superconductor materials that constitute the heterostructures. In addition, the amplitudes of Fano and EIR are higher for the pair of superconductors $(\mathrm{Nb} / \mathrm{BSCCO})$ than the $\left(\mathrm{Rb}_{3} \mathrm{C}_{60} / \mathrm{YBa}_{2} \mathrm{Cu}_{3} \mathrm{O}_{7}\right)$ and $\left(\mathrm{K}_{3} \mathrm{C}_{60} /(\mathrm{BiPb})_{2} \mathrm{Sr}_{2} \mathrm{Ca}_{2} \mathrm{Cu}_{3} \mathrm{O}_{\mathrm{y}}\right)$ ones. This behavior is due to the two coupled oscillator modes that consider the EIR resonance as a special case of Fano resonance happened when the frequencies of strongly and weakly damped oscillators matched ${ }^{33,34}$

Now, we discuss the influence of the operating temperature on both Fano and EIR resonances. Figure 4 shows the dependence of the reflectance on the normalized frequency of the three periodic superconducting PC and for different values of the operating temperature. Here the temperature is set to 2, 4, 6 and $7 \mathrm{~K}$ respectively. For the three structures it is obvious that both Fano and EIR peaks shift to the shorter normalized frequency when the temperature increases. We noted a sharp transition of spectrum of first structure compared to the second and the third one. Furthermore a
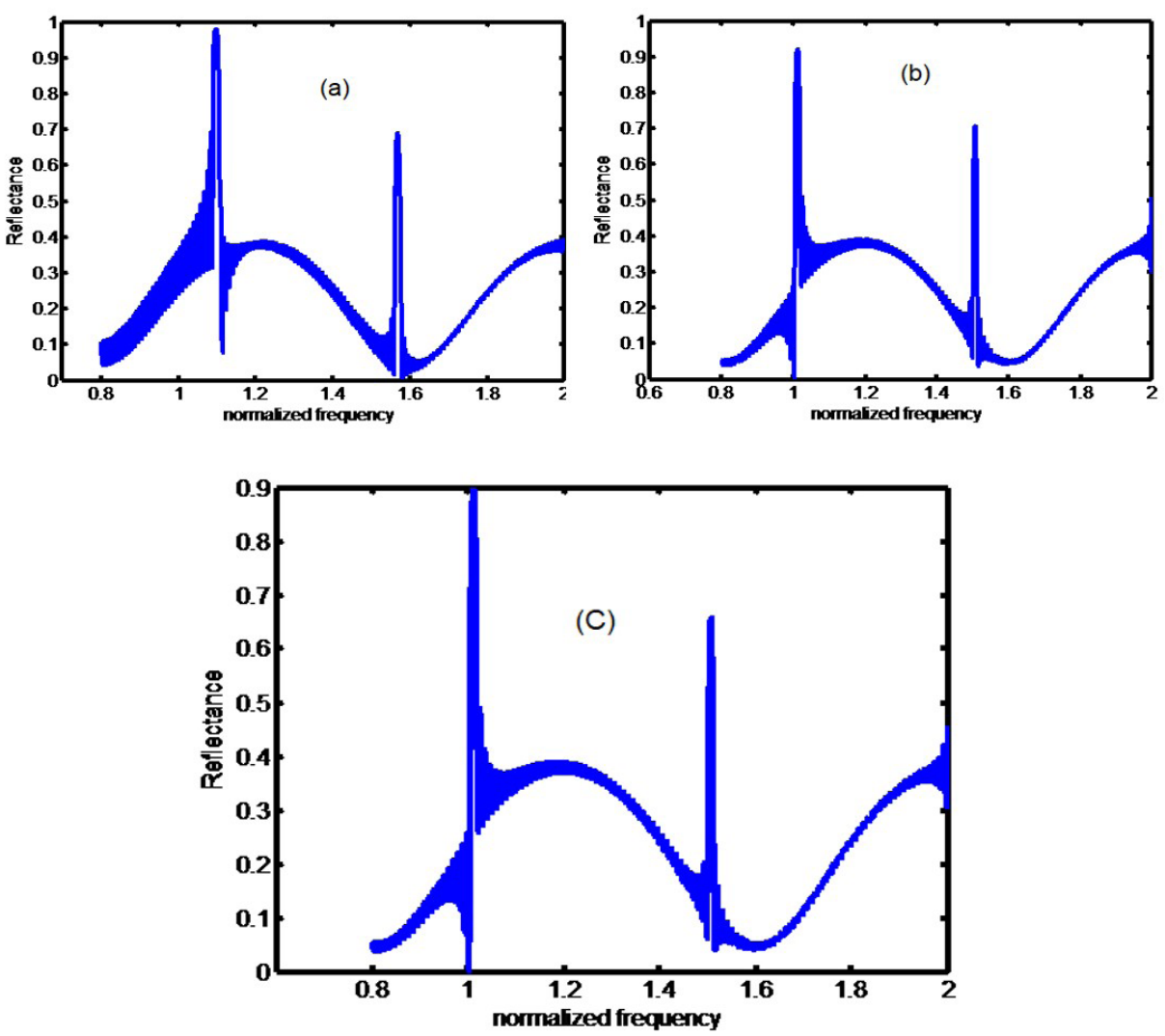

Figure 2. Dependence of the reflectance on the normalized frequency of the three periodic heterostructures: (a) $(\mathrm{Nb} / \mathrm{BSCCO}),(\mathrm{b})\left(\mathrm{Rb} \mathrm{C}_{60}\right.$ $\left./ \mathrm{YBa}_{2} \mathrm{Cu}_{3} \mathrm{O}_{7}\right)$ and (c) $\left(\mathrm{K}_{3} \mathrm{C}_{60} /(\mathrm{BiPb})_{2} \mathrm{Sr}_{2} \mathrm{Ca}_{2} \mathrm{Cu}_{3} \mathrm{O}_{\mathrm{y}}\right)$. 

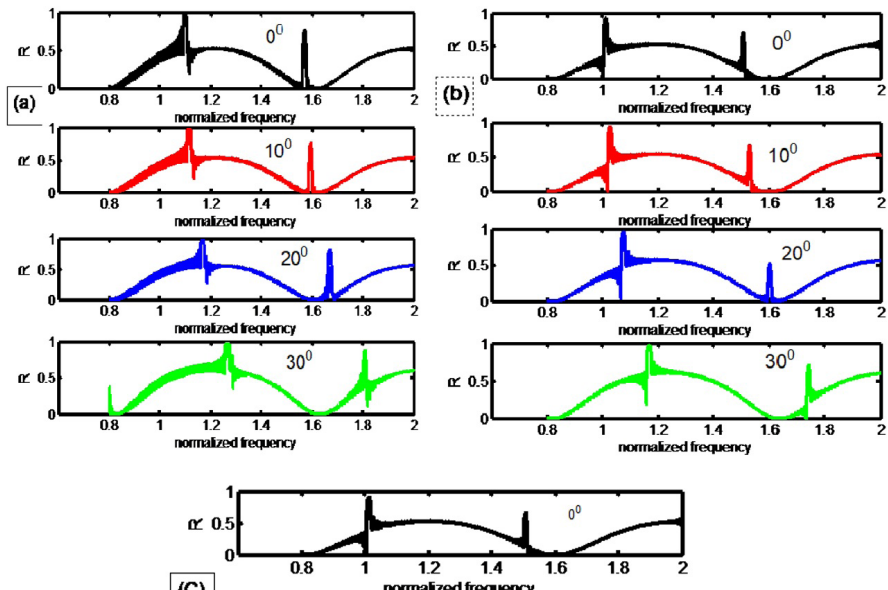

(C)
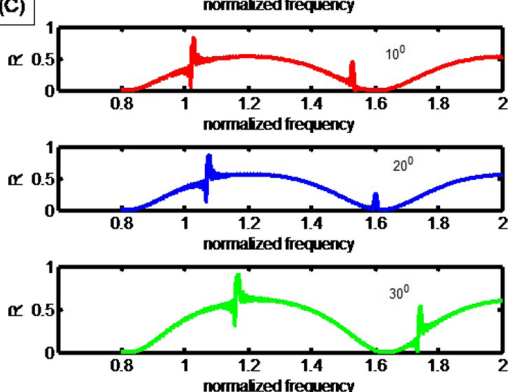

Figure 3. Reflectance spectra versus normalized frequency of the three heterostructures and for different wave incidence angles.
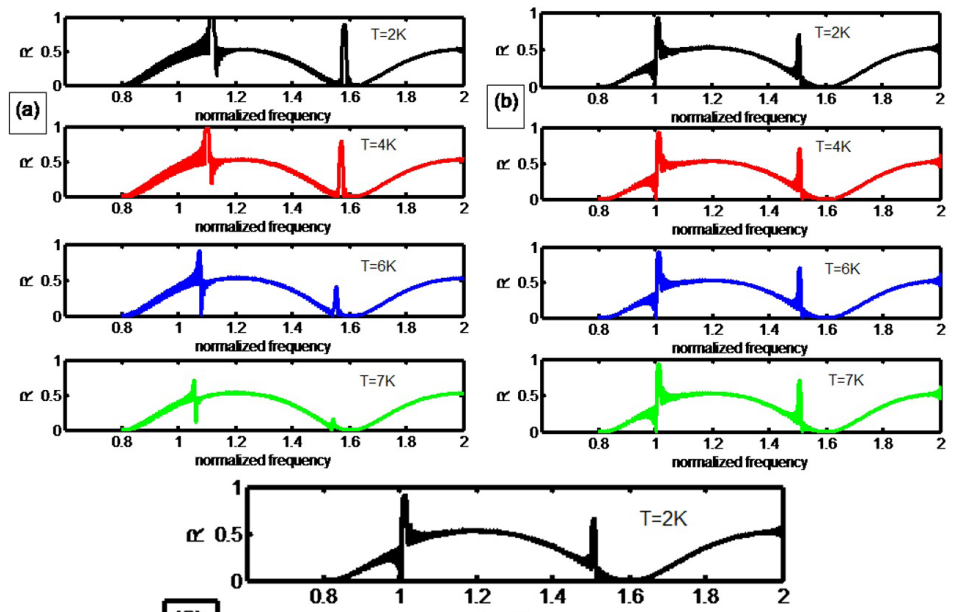

(C)
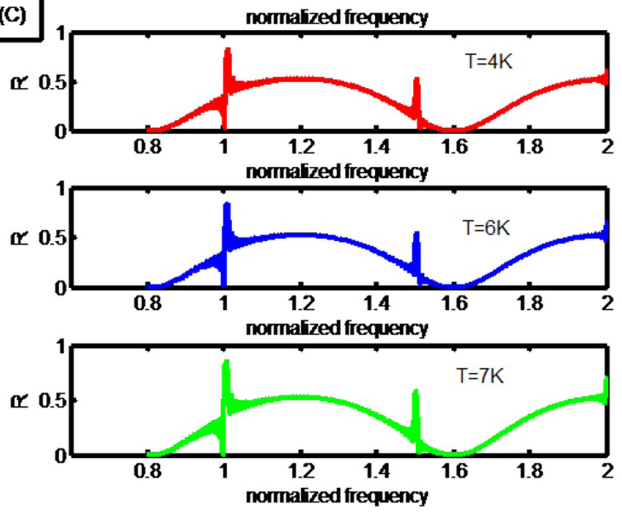

Figure 4. Reflectance spectra versus normalized frequency of the three periodic superconducting PC and for different values of the temperature. 
slowly decrease of the amplitudes of the Fano resonance and a strongly decrease of the EIR one is noticed. Then, the amplitude of the EIR is declined for the (Nb/BSCCO) structure at $\mathrm{T}=7 \mathrm{~K}$. For the second and third structure, the amplitude is slightly affected with the temperature increase.

In this part, we study the effect of change the thickness $d_{1}$ of the superconductor for the three periodic superconducting PC. Figure 5 illustrates the frequency-dependent reflectance for the three pair superconducting PC in TE polarization mode and for different values of $d_{1}\left(d_{1}=70 \mathrm{~nm}, 90 \mathrm{~nm}, 100 \mathrm{~nm}\right.$ and $150 \mathrm{~nm}$ respectively). By increasing the thickness $\mathrm{d}_{1}$, the obtained Fano resonance of three heterostructures shift to the lower frequencies. Whereas, the EIR with symmetrical line shape shift towards the higher frequencies region and it is accompanied by new Fano resonance peaks. It is obvious that from all spectrum the number of Fano resonance peaks depends on thickness $d_{1}$ and on kind of superconductor. With these properties the proposed heterostuctures can serve as multi-optical switching device. Furthermore, it is clear that the amplitude of the Fano resonance and the EIR is higher for the pair $(\mathrm{Nb} / \mathrm{BSCCO})$ than the second and third ones. This first pair constitutes an advantages macroscopic opto-electronic switching in which the damped of oscillation within this behavior is reduced compared to the second and third ones.

For the TE polarized EMWs and for the normal incidence angle the dependence of the reflectance on the refractive index of dielectric cap layer $(1.3,1.6,2$ and 2.5) is illustrated in
Figure 6. By increasing the refractive index of dielectric cap layer, the reflectance spectra are similar in which the position and the amplitude of Fano resonance peaks and the EIRs still the same in the corresponding periodic ( $\mathrm{Nb} / \mathrm{BSCCO})$, and $\left(\mathrm{Rb}_{3} \mathrm{C}_{60} / \mathrm{YBa}_{2} \mathrm{Cu}_{3} \mathrm{O}_{7}\right)$ superconducting PCs. However, in the third $\left(\mathrm{K}_{3} \mathrm{C}_{60} /(\mathrm{BiPb})_{2} \mathrm{Sr}_{2} \mathrm{Ca}_{2} \mathrm{Cu}_{3} \mathrm{O}_{\mathrm{y}}\right)$ superconducting PC the amplitude of EIR decrease progressively when the refractive index of the consider dielectric cap layer increase. This decrease in peak means an incomplete resonance at the defect mode. For $n_{c}=2.5$ the Fano resonance of all proposed superconducting PC becomes symmetrical line shape. In this case, such dielectric cap layer is very important to calibrate the proposed structure by adjusting the characteristics of Fano resonance and EIR peaks.

In Figure 7, the resonance properties are depicted as functions of normalized frequency and for different thicknesses of dielectric cap layer dc (nm). By increasing the dc, the reflectance spectra are similar; in fact the position of all Fano resonances and EIRs are almost the same. Also, all Fano resonances of the three periodic superconducting PCs present two asymmetric resonance peaks. However, the position of given EIR peaks shifted to the lower normalized frequency region when we increase the thicknesses of dielectric cap layer. It should be noted that EIR peaks become with asymmetric shape line when dc exceeds $8 \mathrm{~nm}$ and this phenomenon is due to the increase of the phase shift in the two coupled oscillator mode.
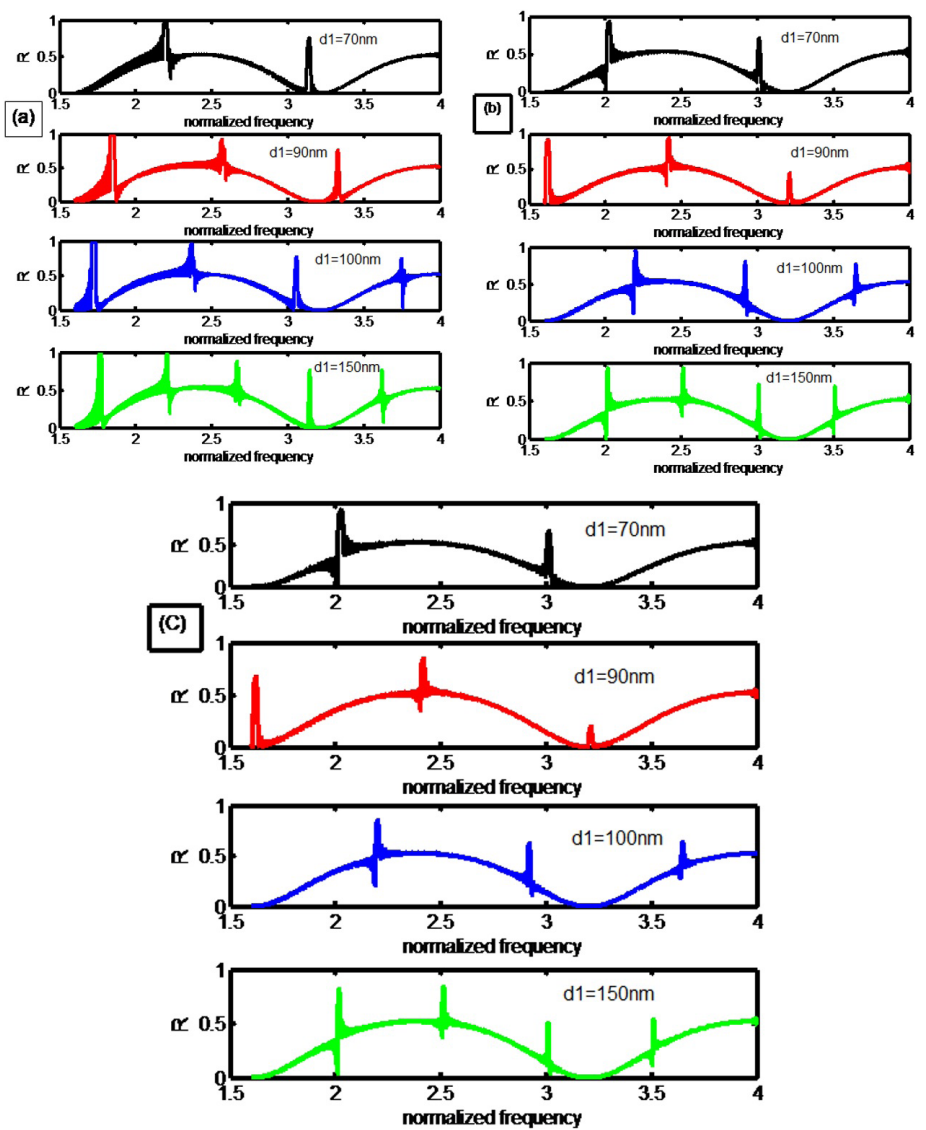

Figure 5. Reflectance spectra versus normalized frequency of the three periodic superconducting PC and for different values of thicknesses $\mathrm{d}_{1}(\mathrm{~nm})$. 


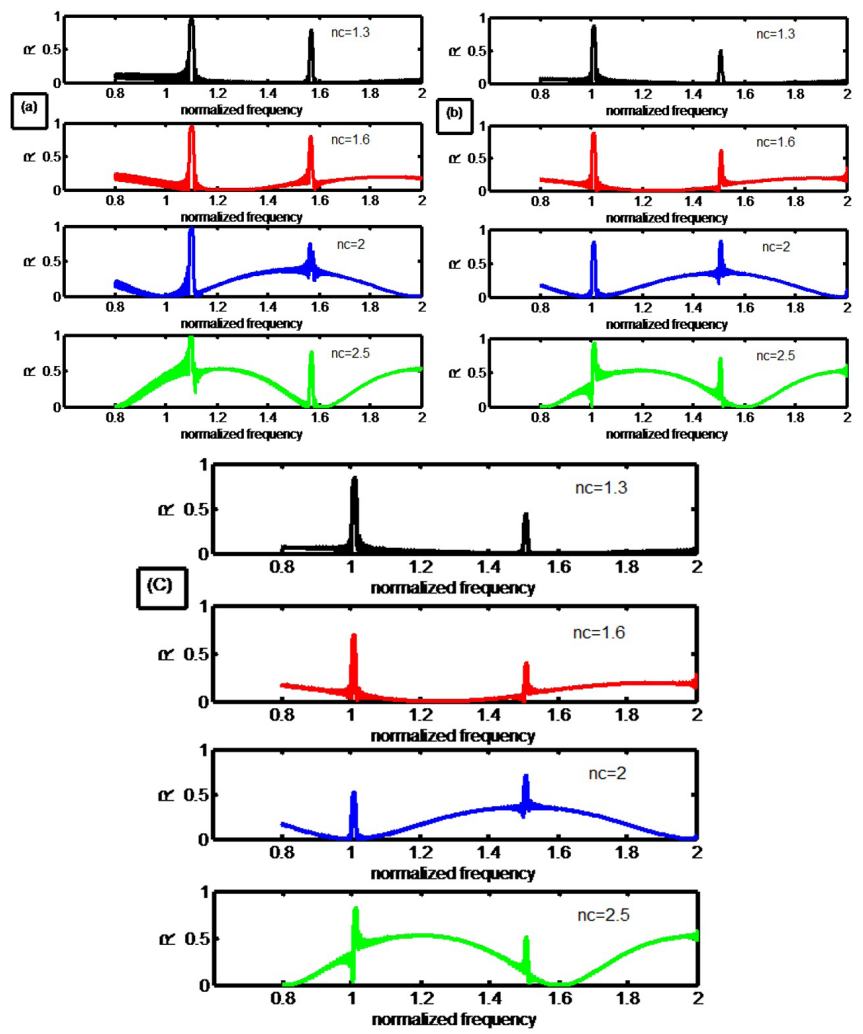

Figure 6. Reflectance spectra versus normalized frequency of the three periodic superconducting PC and for different values of refractive index of the dielectric cap layer.
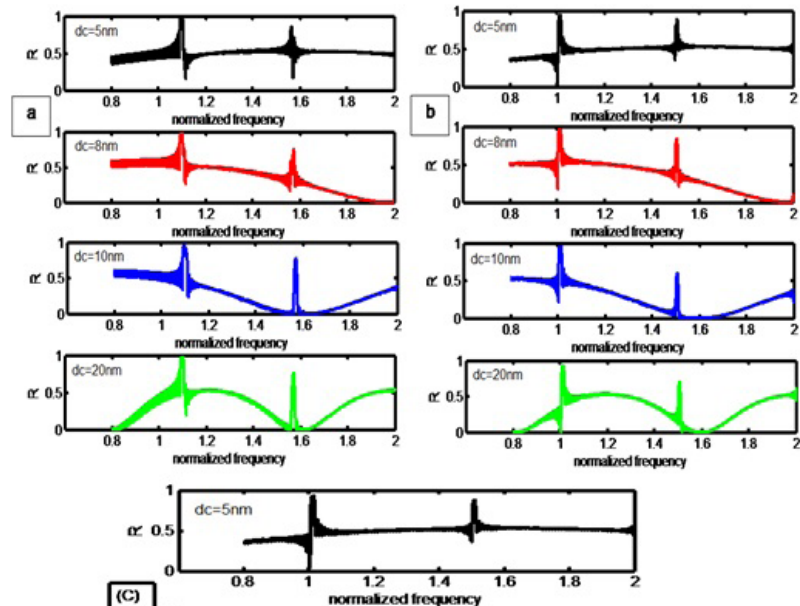

(C)
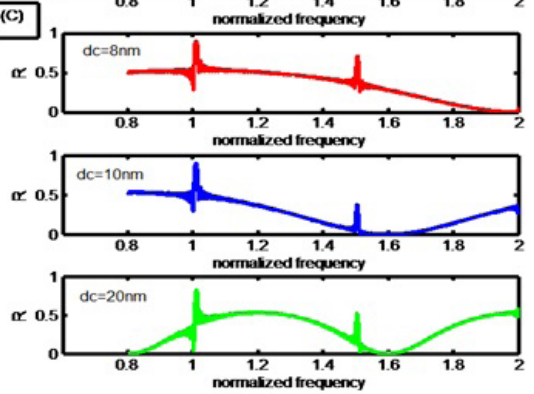

Figure 7. Reflectance spectra versus normalized frequency of the three periodic superconducting PC and for different values of the thickness, dc $(\mathrm{nm})$ of the dielectric cap layer. 


\section{Conclusion}

A comparison study of Fano resonance and EIR properties in different 1D superconducting PCs consisting of the pairs $(\mathrm{Nb} \backslash$ $\mathrm{BSCCO}),\left(\mathrm{Rb}_{3} \mathrm{C}_{60} \mid \mathrm{YBa}_{2} \mathrm{Cu}_{3} \mathrm{O}_{7}\right)$ and $\left(\mathrm{K}_{3} \mathrm{C}_{60} \backslash(\mathrm{BiPb})_{2} \mathrm{Sr}_{2} \mathrm{Ca}_{2} \mathrm{Cu}_{3} \mathrm{O}_{\mathrm{y}}\right)$ superconducting materials are investigated. All suggested structures exhibit a tunable Fano resonances and EIR resonance peak accompanied by asymmetrical line shape. These resonances are very sensitive to the thicknesses and the refractive index of dielectric cap layer, the kind of materials, the wave incidence angle and the temperature. The $(\mathrm{Nb} / \mathrm{BSCCO})$ structure showed the best-reported results in which amplitude of given Fano resonance and EIR is higher than others structures. Furthermore, the obtained spectra are tunable by adjusting the value of the optical parameters. As a result, a good response with maximum peaks of reflection is given by the $(\mathrm{Nb} / \mathrm{BSCCO})$ structure. This structure can find application in the field of optical communication and it can be used as optical switcher.

\section{Acknowledgements}

The authors are thankful to the Deanship of Scientific Research- Research Center at King Khalid University in Saudi Arabia for funding this research (code number: GRP/91/42).

\section{References}

1. Yablonovitch E. Inhibited spontaneous emission in solid-state physics and electronics. Phys Rev Lett. 1987;58(20):2059-62.

2. Yablonovitch E. Photonic band-gap structures. J Opt Soc Am B. 1993;10:283-95.

3. Chen X-D, Shi F-L, Liu H, Lu J-C, Deng W-M, Dai J-Y, et al. Tunable electromagnetic flow control in valley photonic crystal waveguides. Phys Rev Appl. 2018;10:044002.

4. Aly AH, Mohamed D, Zaky ZA, Matar ZS, Abd El-Gawaad NS, Shalaby AS, et al. Mohaseb, novel biosensor detection of tuberculosis based on photonic band Gap materials. Mater Res. 2021;24(3):e20200483. http://dx.doi.org/10.1590/1980-5373MR-2020-0483.

5. Khateib F, Mehaney A, Amin RM, Aly AH. Ultra-sensitive acoustic biosensor based on a 1D phononic crystal. Phys Scr. 2020;95:075704.

6. Aly AH, Mohamed D, Mohaseb M-A, Abd El-Gawaad NS, Trabelsi Y. Biophotonic sensor for the detection of creatinine concentration in blood serum based on 1D photonic crystal. RSC Advances. 2020;10:31765-72.

7. Wiesmann C, Bergenek K, Linder N, Schwarz UT. Photonic crystal LEDs - designing light extraction. Laser Photonics Rev. 2009;3:262-86.

8. Nozaki K, Baba T. Quasiperiodic photonic crystal microcavity lasers. Appl Phys Lett. 2004;84:4875-7.

9. Sayed H, Aly AH. Salinity optical sensor by using twodimensional Photonic crystals: computational study. Mater Sci Eng B. 2021;269:115169.

10. Inui C, Tsuge Y, Kura H, Fujihara S, Shiratori S, Sato T. Preparation of one-dimensional photonic crystals by sol-gel process for magneto-optical materials. Thin Solid Films. 2007;516:2454-9.

11. Zaky ZA, Aly AH. Theoretical study of a tunable low-temperature photonic crystal sensor using dielectric-superconductor nanocomposite layers. J Supercond Nov Magn. 2020;33:2983-90.

12. Aly AH, Sabra W. Superconductor-semiconductor metamaterial photonic crystals. J Supercond Nov Magn. 2016;29:1981-6.

13. Aly AH, Ryu SW, Hsu HT, Wu CJ. THz transmittance in one-dimensional superconducting nanomaterial-dielectric superlattice. Mater Chem Phys. 2009;113:382-4.
14. Trabelsi Y. Output multichannel optical filter based on hybrid photonic quasicrystals containing a high-Tc superconductor. Photon Nanostructures. 2019;36:100724.

15. Wu J, Gao J. Analysis of temperature-dependent optical properties in $1 \mathrm{D}$ ternary superconducting photonic crystal with mirror symmetry. J Supercond Nov Magn. 2015;28:1971-6.

16. Segovia-Chaves F, Vinck-Posada H, Trabelsi Y, Ben Ali N. Transmittance spectrum in a one-dimensional photonic crystal with Fibonacci sequence superconductor-semiconductor. Optik (Stuttg). 2020;217:164803.

17. Trabelsi Y, Ali NB, Aly AH, Mounir K. Tunable high Tc superconducting photonic band gap resonators based on hybrid quasi-periodic multilayered stacks. Physica C: Supercond. 2020;576:1353706.

18. Aly AH, Abdel Ghany SES, Ghany A, Kamal BM, Vigneswaran D. Theoretical studies of hybrid multifunctional $\mathrm{YaBa}_{2} \mathrm{Cu}_{3} \mathrm{O}_{7}$ photonic crystals within visible and infra-red regions. Ceram Int. 2020;46(1):365-9.

19. Athe P, Srivastava $S$. Tunable fano resonance in one-dimensional superconducting photonic crystal. J Supercond Nov Magn. 2015;28:2331-6.

20. Song J, Proietti Zaccaria R, Yu MB, Sun XW. Tunable Fano resonance in photonic crystal slabs. Opt Express. 2006;14(19):881226. PMid: 19529263.

21. Singh R, Al-Naib I-A, Koch M, Zhang W. Sharp Fano resonances in THz metamaterials. Opt Express. 2011;19(7):6312-9. PMid:21451657.

22. Dong G, Wang Y, Zhang X. High-contrast and low-power alloptical switch using Fano resonance based on a silicon nanobeam cavity. Opt Lett. 2018;43(24):5977-80. PMid:30547984.

23. Zhao W, Leng X, Jiang Y. Fano resonance in all-dielectric binary nanodisk array realizing optical filter with efficient linewidth tuning. Opt Express. 2015;23(5):6858-66. PMid:25836905.

24. Cao T, Zhang L, Xiao Z-P, Huang H. Enhancement and tunability of Fano resonance in symmetric multilayer metamaterials at optical regime. J Phys D Appl Phys. 2013;46:395103.

25. Luk'yanchuk B, Zheludev N-I, Maier S-A, Halas N-J, Nordlander P, Giessen H, et al. The Fano resonance in plasmonic nanostructures and metamaterials. Nat Mater. 2010;9(9):707-15. PMid:20733610.

26. Aly AH, Mohamed D, Mohaseb MA. Metamaterial control of hybrid multifunctional High-Tc superconducting photonic crystals for 1D Quasi-periodic structure potential applications. Mater Res. 2020;23(3):e20190695.

27. Aly AH, Mohamed D, Elsayed HA, Mehaney A. Fano resonance by means of the one-dimensional superconductor photonic crystals. J Supercond Nov Magn. 2018;31:3827-33.

28. Schöck M, Sürgers C, Löhneysen Hv. Superconducting and magnetic properties of $\mathrm{Nb} / \mathrm{Pd} 1-\mathrm{xFex} / \mathrm{Nb}$ triple layers. Eur Phys J B Cond Matter Complex Syst. 2000;14:1-10.

29. Aly AH, Mohamed D. BSCCO/SrTiO3 one dimensional superconducting photonic crystal for many applications. J Supercond Nov Magn. 28(1):1699-703.

30. Lee H-M, Wu J-C. Transmittance spectra in one-dimensional superconductor-dielectric photonic crystal. J Appl Phys. 2010;107:09E149.

31. Srivastava SK. Study of defect modes in 1d photonic crystal structure containing high and low Tc superconductor as a defect layer. J Supercond Nov Magn. 2014;27:101-14.

32. Holesinger TG, Bingert JF, Willis JO, Li Q, Parrella RD, Teplitsky $\mathrm{MD}$, et al. Structural and compositional defects in high-J/sub c/ Bi-2223 tapes. IEEE Trans Appl Supercond. 1999;9:2.

33. Yang X, Yu M, Kwong D-L, Wong CW. All-optical analog to electromagnetically induced transparency in multiple coupled photonic crystal cavities. Phys Rev Lett. 2009;102(17):173902.

34. Yang Y, Kravchenko II, Briggs DP, Valentine J. All dielectric metasurface analogue of electromagnetically induced transparency. Nat Commun. 2014;5:5753. 